\title{
One Hamming Distance Deviation Matching Approach For Iris Recognition
}

\author{
Lv Hanfei and Jiang Congfeng \\ Department of Information management Zhejiang Vocational Policy Academy \\ Hangzhou, Zhejiang Province, China \\ School of Computer Science Hangzhou Dianzi University Hangzhou, Zhejiang \\ Province, China \\ lhf_b@sina.com cjiang@hdu.edu.cn
}

\begin{abstract}
In this paper we propose a Hamming Distance Deviation Matching Approach (HDDMA) for Iris recognition. Our HDDMA approach is different from the traditional iris matching method based on Hamming Distance. Firstly we use the odd symmetry Gabor filters with single frequency and two directions to extract iris edge information. Secondly we use zero-crossing detecting method to encode the filtering results. Finally we construct the HDDMA parameter for iris matching. Comparison experiments between the traditional Hamming Distance matching method and the proposed HDDMA are conducted on five iris datasets. The experimental results show that the equal error rate and the correct recognition ratio of the HDDMA are better than those of the traditional Hamming Distance matching method consistently in all iris datasets and the HDDMA has strong anti-eyelid and eyelash noise capability .
\end{abstract}

Keywords: Feature matching, Hamming Distance Deviation Matching Approach, Iris recognition

\section{Introduction}

Iris recognition technology is an ideal identification approach because the texture of the iris is unique and stable. It has two outstanding advantage-high accuracy and high security, and it has important application prospects [1,2].

We list them represent iris feature extraction and matching algorithm as below:

Daugman [3] has presented one two-dimensional (2D) Gabor filter based method for the iris identification system and he used Hamming Distance method for iris matching. Wildes, et al., [4] used Laplacian pyramid for efficient implementation of gradient-based iris segmentation [5]. Boles have presented fine-to-coarse approximation at diverse resolution levels that are based on zero-crossing representation from the wavelet transform decomposition. He uses European distance method for iris matching. Ma, et al., [7] utilized multi-scale band-pass decomposition and evaluated comparative performance from prior methods.

2D Gabor filter has the frequency and direction information. It is the main approach for the extraction of iris feature. Many researchers raised various iris features extracting method and encoding method. But most of them use the traditional Hamming Distance matching method. Therefore they cannot improve the recognition ratio further more. We not only construct Gabor filter with single frequency and two directions, but also raised HDDMA for iris matching. Experiments show it can promote the recognition ratio. 


\section{Iris Pre-Processing}

In this paper we use rapid iris localization based on method of iterative pixel ratio to cirque area. We adopt the Daugman's elastic model. From transformation of coordinates the circle iris image changed to $512 \times 64$ matrix image in normalization. Then we trunk the 1/4 image of top right corner to eliminate the noise. Finally we use histogram equalization method to strength the image.

\section{Iris Feature Extraction}

\subsection{Filter Selection}

We use 2D Gabor filter:

$$
\begin{aligned}
& G(x, y)=\frac{1}{2 \pi \rho_{x} \rho_{y}} \exp \left(2 \pi i f x^{\prime}\right) \exp \left(-\frac{1}{2}\left(\frac{x^{\prime^{2}}}{\rho_{x}{ }^{2}}+\frac{y^{\prime^{\prime 2}}}{\rho_{y}{ }^{2}}\right)\right) \\
& x^{\prime}=y \sin d+x \cos d \\
& y^{\prime}=y \cos d-x \sin d
\end{aligned}
$$

Here $\rho_{x}$ and $\rho_{y}$ are dimension parameter and $f$ stand for the radial frequency. Parameter $d$ stands for the direction of the Gabor filter. The 2D Gabor filter consists of real part even symmetry Gabor filter and imaginary part odd symmetry filter. We list the odd symmetry Gabor filter as below:

$$
G_{0}(x, y)=\frac{1}{2 \pi \rho_{x} \rho_{y}} \exp \left(2 \pi f x^{\prime}\right) \exp \left(-\frac{1}{2}\left(\frac{x^{\prime^{2}}}{\rho_{x}{ }^{2}}+\frac{y^{\prime^{2}}}{\rho_{y}{ }^{2}}\right)\right)
$$

The odd symmetry Gabor filter has a edge examination feature and can classify the contrary texture change. Especially, it is free from strength of illumination. The odd symmetry Gabor filter is suitable for extraction of iris edge feature.

\subsubsection{Direction Selection}

We use parameter $d$ to represent the direction of the Gabor filter. 2D Gabor complex filter consists of two filters. One is real part even symmetry Gabor filter, another is imaginary part odd symmetry Gabor filter.

We construct eight Gabor filter with four direction. When $d=0^{\circ}, d=45^{\circ}, d=90^{\circ}, d=135^{\circ}$, its 3D image and gray image are shown as Figure 1.
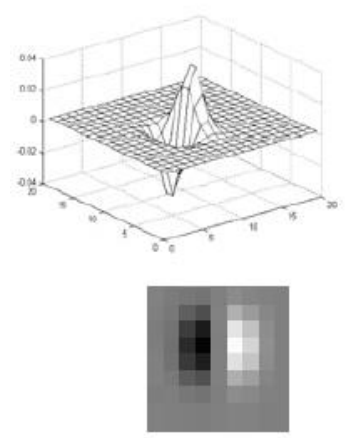

$\mathrm{d}=0^{\circ}$
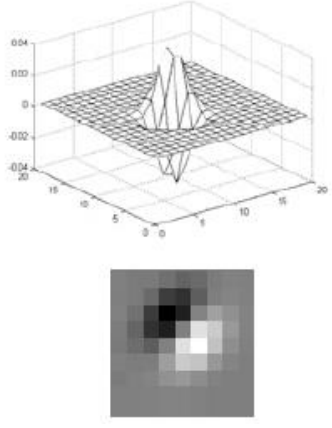

$\mathrm{d}=45^{\circ}$
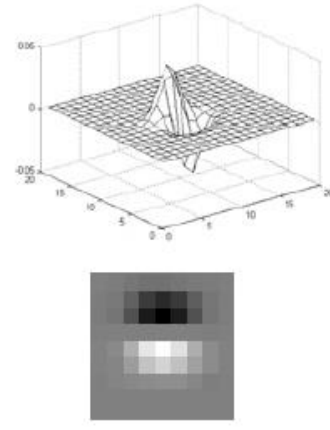

$\mathrm{d}=90^{\circ}$
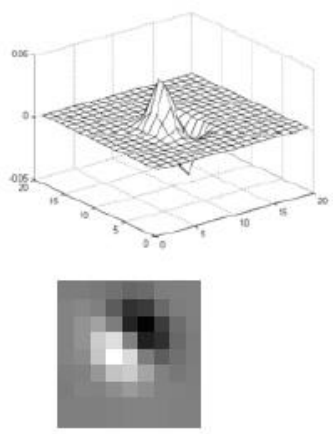

$\mathrm{d}=135^{\circ}$

(a) odd symmetry Gabor filter 

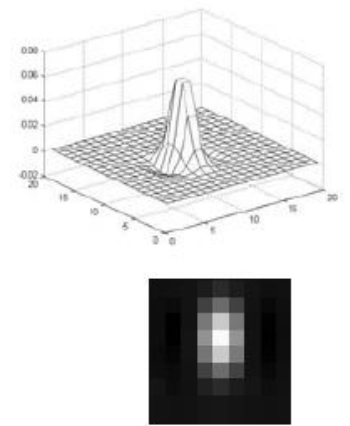

$\mathrm{d}=0^{\circ}$
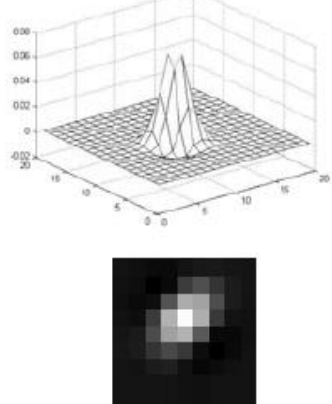

$\mathrm{d}=45^{\circ}$
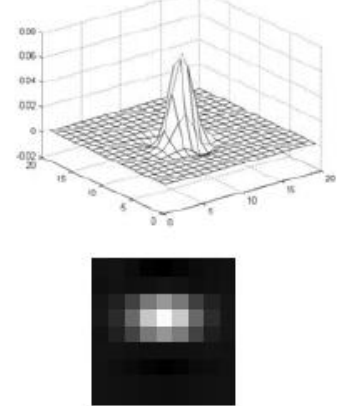

$\mathrm{d}=90^{\circ}$
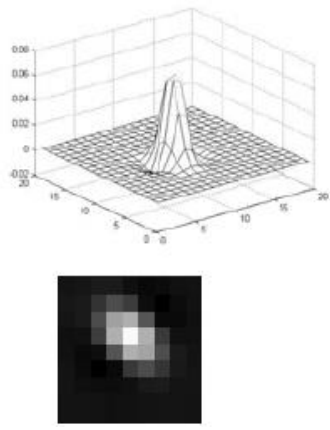

$\mathrm{d}=135^{\circ}$

(b) even symmetry Gabor filter

Figure 1. 2D Gabor Filter

We show the direction and the edge image in Figure 2:

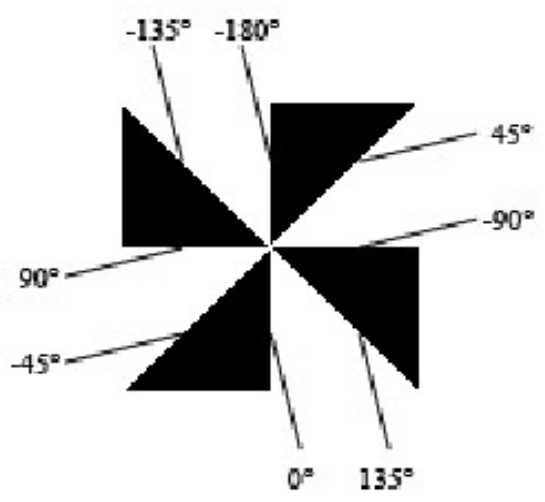

Figure 2. Direction and Edge Image

We get the filter result in Figure 3. Here we set frequency $f$ to 0.125 , and $\rho_{x}=\rho_{y}=1.414$.

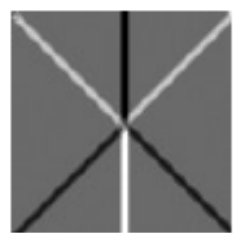

$\mathrm{d}=0^{\circ}$

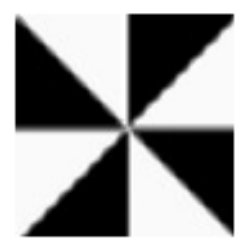

$\mathrm{d}=0^{\circ}$

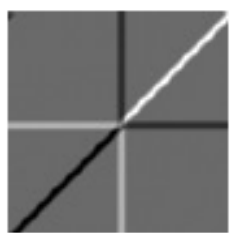

$\mathrm{d}=45^{\circ}$

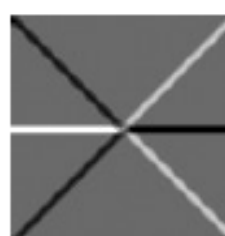

$\mathrm{d}=90^{\circ}$

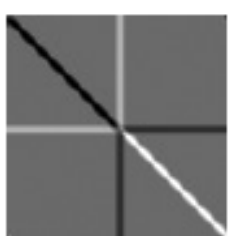

$\mathrm{d}=135^{\circ}$

(a) odd symmetry Gabor filter

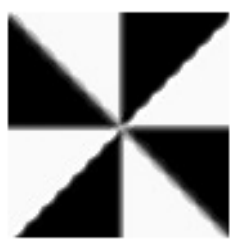

$\mathrm{d}=45^{\circ}$

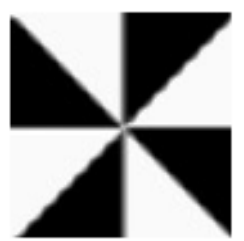

$\mathrm{d}=90^{\circ}$

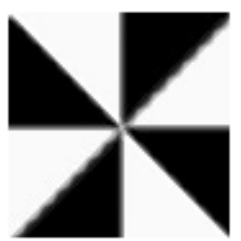

$\mathrm{d}=135^{\circ}$

(b) even symmetry Gabor filter

Figure 3. Filtering Result 
From the waveform and the filtering result we find that the odd symmetry Gabor filter suits to extract the iris texture features better than the even symmetry Gabor filter. The odd symmetry Gabor filter has three advantages:

(a) The odd symmetry Gabor filter is a gradient detector, and it can effectively detect the iris image edge. From the filtering result we know if we set direction $\mathrm{d}$ for one odd symmetry Gabor filter, the boundary response gets the maximum value in direction $\mathrm{d}$, then direction $\mathrm{d} \pm 45^{\circ}$. The boundary response is 0 in direction $\mathrm{d} \pm 90^{\circ}$. The odd symmetry Gabor filter can provide good direction selectivity. On the contrary, the even symmetry Gabor filter cannot effectively detect the iris image edge information.

(b) The odd symmetry Gabor filter can distinguish in the direction opposite texture with odd symmetry property. From filtering result of the odd symmetry Gabor filter we know the boundary response get the same absolute value for direction $\mathrm{d}$ and $\mathrm{d}-180^{\circ}$. Here one is negative, one is positive. We yield the same conclusion for direction $\mathrm{d} \pm 45^{\circ}$ and $\mathrm{d} \pm 45^{\circ}-180^{\circ}$. From the filtering result of the even symmetry Gabor filter we know the boundary response get the same value for direction $\mathrm{d}$ and $\mathrm{d}-180^{\circ}$. We yield the same conclusion for direction $\mathrm{d} \pm 45^{\circ}$ and $\mathrm{d} \pm 45^{\circ}-180^{\circ}$. The even symmetry Gabor filter cannot distinguish in the direction opposite texture with even symmetry property.

(c) The odd symmetry Gabor filter is a strict band pass filter. It is not affected by the DC and low frequency information brought by illumination changes or interferes with uneven illumination. However, the even symmetry Gabor filter is not a strict band pass filter. It allows some DC and low frequency information. Therefore the even symmetry Gabor filter is affected by illumination changes or interfere with uneven illumination when it is used for iris texture extraction.

In conclusion, the odd symmetry Gabor filter is suitable for the iris feature extraction because it has excellent boundary detection, direction selectivity, odd symmetry property and band pass property.

The parameter $d$ decides the direction of filter, figure 1 show $0^{\circ} \sim 135^{\circ}$ total 4 direction Gabor filters result. We found the Gabor filter with different direction angle $\mathrm{d}$ all has response from $\mathrm{d}-90^{\circ}$ to $\mathrm{d}+90^{\circ}$ directions. We got the strongest response from direction $\mathrm{d}$. And the response decrease gradually when we rotate from $d$ to $d \pm 90^{\circ}$. In direction $d \pm 90^{\circ}$ the response decreases to zero.

We select two filters from different direction: one is $0^{\circ}$, another is $90^{\circ}$. The $0^{\circ}$ filter's detection scope is $\left(-90^{\circ},+90^{\circ}\right)$, and the $90^{\circ}$ filter detection scopes is $\left(0^{\circ},+180^{\circ}\right)$. Considering the odd symmetry, the whole scope of two filters can cover the total $360^{\circ}$.

\subsubsection{Frequency Selection}

Here $f$ is the radial frequency of the filter. The 2D Gabor filters with different frequency has different texture response. We construct four image texture sizes to Figure out the 2D odd symmetry Gabor filter frequency selectivity. In Figure 4 they are $4 \times 4,8 \times 8,16 \times 16,32 \times 32$ images below. 


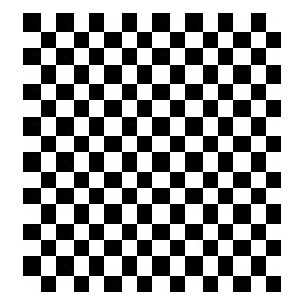

(a) $4 \times 4$

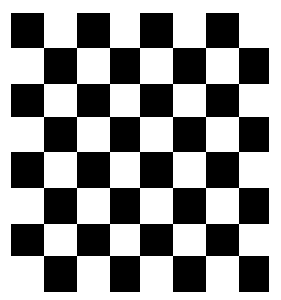

(b) $8 \times 8$

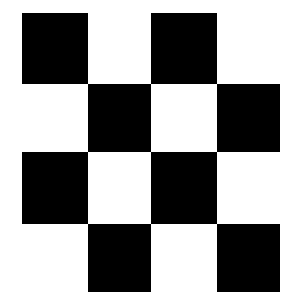

(c) $16 \times 16$

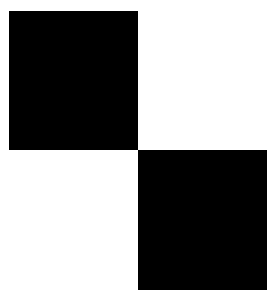

(d) $32 \times 32$

Figure 4. Different Size Texture Image

Here we set direction paramter $\mathrm{d}=0^{\circ}$ without loss of generality. We use odd symmetry Gabor filter to extract the texture with different frequency $0.03125,0.0625,0.125$ and 0.25 . We set $\rho_{x}=\rho_{y}=5.657,{ }^{\rho_{x}}={ }^{\rho_{y}}=2.828,{ }^{\rho_{x}}={ }^{\rho_{y}}=1.414$ and ${ }^{\rho_{x}}=\rho_{y}=0.707$ for different frequency to guaranty that the filter contains only one wavelength. We show the $2 \mathrm{D}$ odd symmetry Gabor filter with different frequency in Figure 5 as below:

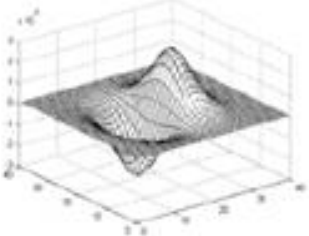

(a) $f=0.03125$

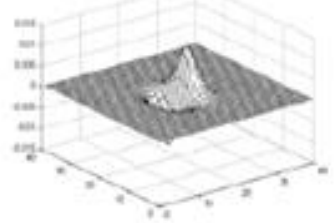

(b) $f=0.625$

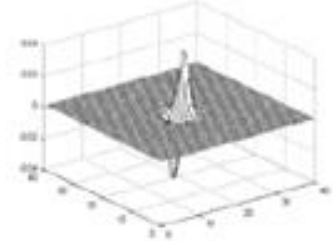

(c) $f=0.125$

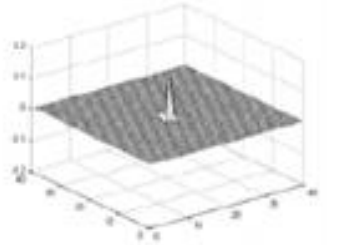

(d) $f=0.25$

Figure 5. 2D Odd Symmetry Gabor Filter with Different Frequency

The filtering result is shown in Figure 6 as below.
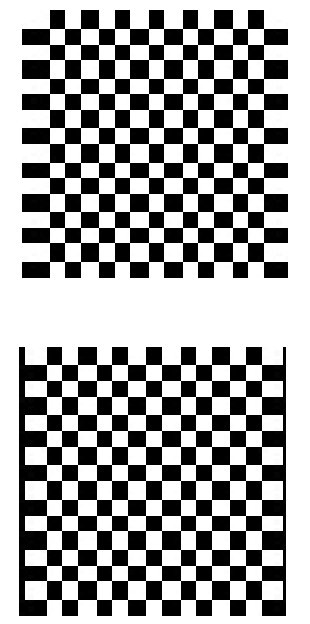
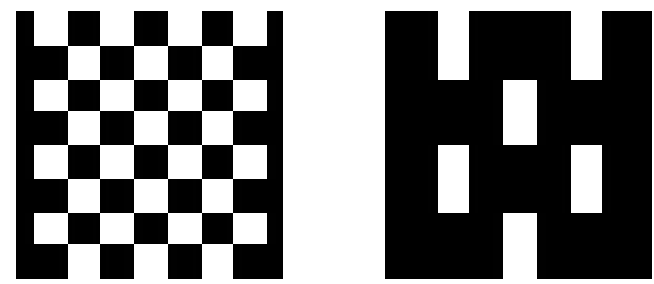

(a) $f=0.03125$
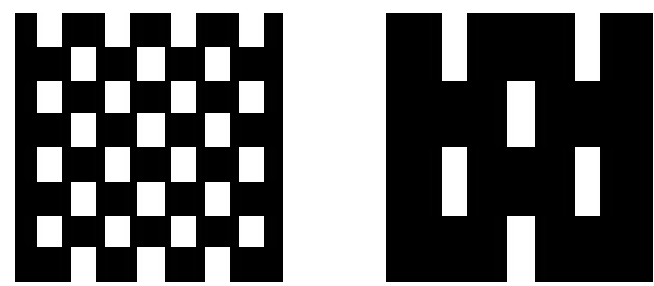

(b) $f=0.625$
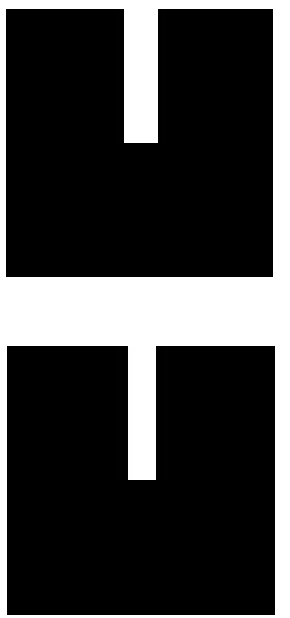

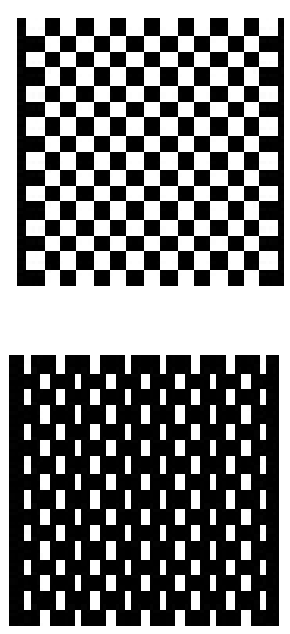
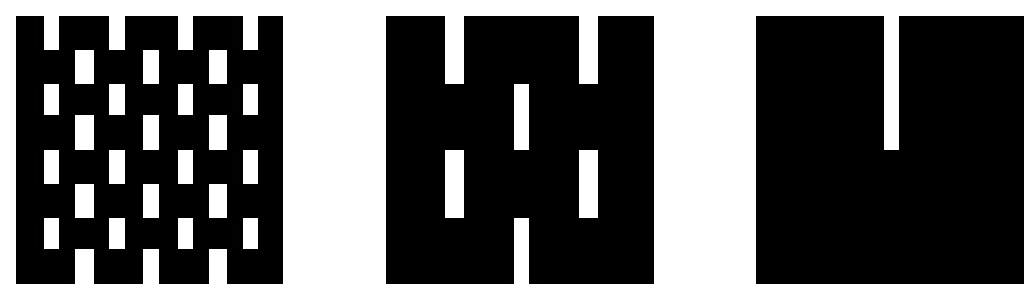

(c) $f=0.125$
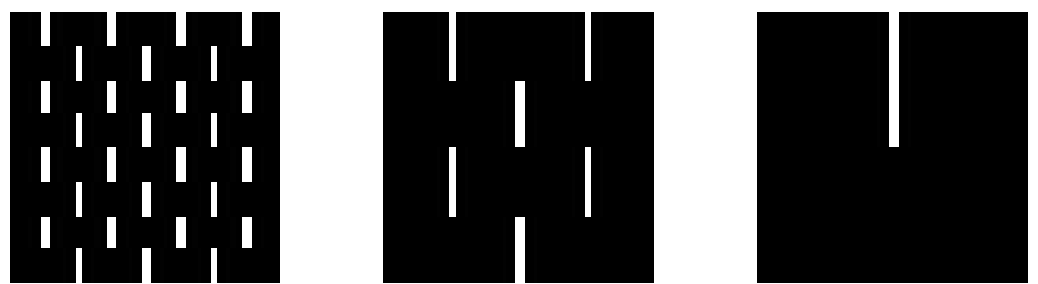

(d) $f=0.25$

\section{Figure 6. Filter Result with Different Frequency}

Because the odd symmetry Gabor filters is an edge detector it can detect the edge texture in direction $0^{\circ}$. The frequency is larger; the edge width detected is smaller. The filter cannot effectively detect the $8 \times 8$ texture or smaller texture with frequency 0.03125 . The filter cannot effectively detect the $4 \times 4$ texture or smaller texture with frequency 0.0625 and 0.125 . And the filter can effectively detect the $2 \times 2$ texture or larger texture with frequency 0.25 . Therefore the odd symmetry Gabor filter has good frequency selectivity.

Here the parameter $f$ stands for the radial frequency, and the filter with frequency $f$ can examine the texture with frequency smaller than $f$. We are only interested to the iris texture of edge information; therefore we choose the single frequency filter rather than the multifrequency filter. The value of frequency $f$ should not be too high or too low. After the trial test we set the value of frequency $f$ to 0.125 . It can detect all the texture edge with width larger than 4 .

\subsubsection{Dimension Selection}

The dimension parameter $\rho_{x}$ and $\rho_{y}$ respectively decide the $\mathrm{x}$ and y direction range of the Gabor filter. Iris has no special texture direction, therefore $\rho_{x}=\rho_{y}=\rho$. The odd symmetry filter is edge extraction filter, and the effect of edge detection is the best when the frequency is one wavelength. Here $\rho$ will be changed with the change of frequency f. The Gaussian window function of Gabor filter is listed as below:

$$
g(x, y)=\frac{1}{2 \pi \rho^{2}} \exp \left(-\frac{x^{2}+y^{2}}{2 \rho^{2}}\right)
$$




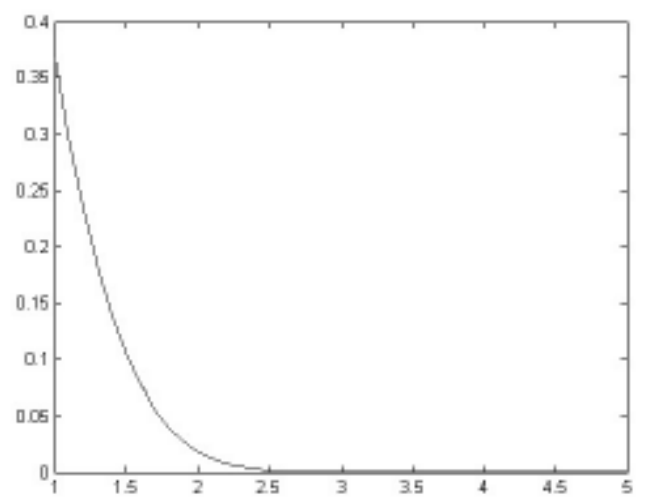

Figure 7. $e^{-N^{2}}$ Function Curve

We show the function curve in Figure 7. Because when $\mathrm{N}=2 \sqrt{2}$ the exponential function $e^{-N^{2}}$ nearly equals to $100 \%$. ( $\left.e^{-8}=99.966 \%\right)$ It is suitable to use circle area to represent the local effective coverage.

$$
\frac{x^{2}+y^{2}}{2 \rho^{2}}=N^{2}=8
$$

One wavelength filter we can get the relational expression:

$$
\frac{1}{f}=2 \times 2 \sqrt{2} \times \sqrt{2} \rho \text {, When } f=0.125, \rho=\frac{1}{8 f}=1 .
$$

\subsection{Building Filter Set}

In summary, we construct one odd symmetry Gabor filter with single frequency and two directions. The frequency $f$ of the filter is 0.125 , the direction $\mathrm{d}$ is $0^{\circ}$ and $90^{\circ}$, Dimension parameter $\rho_{x}=\rho_{y}=\rho=1$.

\section{The Zero-Crossing Coding Approach}

We use the zero-crossing coding method. Once the value of one bit is larger than 0 , we set the bit value 1 . Or we set the bit value 0 . We can use 2 bits to represent each pixel. We adopt the sampling method to construct the 2048 bits code length. There are 128 bits in horizontal direction and 8 bits in vertical direction.

\section{Iris Matching Method}

\subsection{Traditional Matching Methods}

The traditional iris matching method is Hamming Distance method.

$H D=\frac{1}{M} \sum_{i=1}^{M} A_{i} \oplus B_{i}$

$A_{i}$ and $B_{i}$ are two different iris encoding. $\oplus$ is EOR calculation. $M$ is the total bits. The 
iris normalization only corrects the handle the translation and zooming. It cannot correct the iris rotation for the reason of the head movement. Iris matching method usually uses horizontal displacement to eliminate the rotation. In general we got the minimum $\mathrm{HD}_{\min }$ when the best iris match happened.

$\mathrm{HD}_{\min }=\min \left(\mathrm{HD}_{\mathrm{i}}\right), \mathrm{i}=-\mathrm{n},-\mathrm{n}+1, \ldots, \mathrm{n}$,

Here $\mathrm{HD}_{\mathrm{i}}$ means the $\mathrm{HD}$ value when we horizontal displace $\mathrm{i}$ bits. + and - means the direction. From right to left is -, from left to right is + . And $n$ is the largest offset of the pixel bits.

\subsection{HDDMA Iris Matching Method}

The traditional iris matching method use $\mathrm{HD}_{\min }$ to do the shift match basis, but it does not consider the whole process. We show the shift curve of two matching approach in Figure 8. We found the different iris fluctuates smoothly but the same iris fluctuates vigorously in the best matching place.

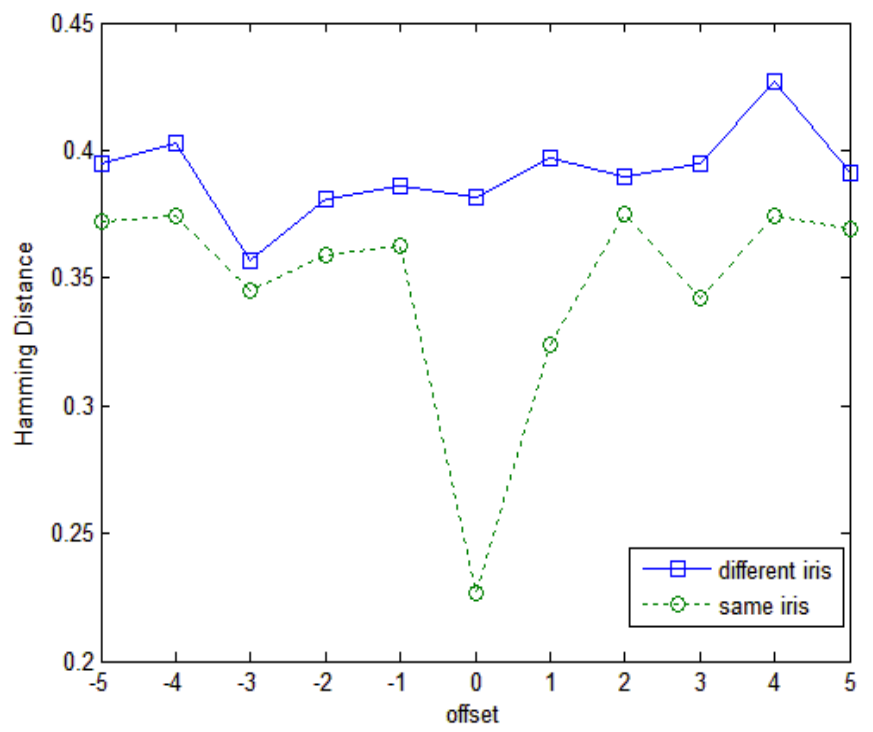

Figure 8. Offset HD Curve

Deviation is defined as the average number of distance from the data to the average data. It reflects the wavy degree of the curve. And the Match of move a HD curve can see matching of a different iris curve. We give the definition of the HDD parameter as below:

$$
H D D=\sqrt{\frac{\sum_{i=-n}^{n}\left(H D_{i}-H D_{\text {min }}\right) 2}{2 n+1}}
$$

Here $\mathrm{n}$ means the offset. $\mathrm{HD}_{\min }$ is the $\mathrm{HD}$ value in the best matching place. $\mathrm{HDD}$ can be iris matching parameter because the same iris got much bigger value than the different iris near the best matching place. 


\section{Experiments and the Result Analysis}

\subsection{Experiment Environment and Iris Identify Standard}

We use 5 iris databases in CASIA (http://www.cbsr.ia.ac.cn/IrisDatabase.htm) They are CASIA-V1.0, CASIA-V2.0-Device1, CASIA-V2.0-Device2, CASIA-V3.0-Interval and CASIA-V3.0-Twins. In general, several parameters include FRR (False Rate of Reject), FAR(False Accept Rate), EER( Equal Error Rate) and CRR(Correct Recognition Rate) are used to evaluate the performance of the algorithm. Our target is to get low EER and high CRR at the same time. It means great performance.

\subsection{Experiment Comparison}

We have done the compare experiment in 5 iris databases. They are CASIA-V1.0, CASIAV2.0-Device1, CASIA-V2.0-Device2, CASIA-V3.0-Interval and CASIA-V3.0-Twins. The first step is pre-processing, the second step is feature extraction, the third step is the iris encoding, and the last step is the match. Here we focused on the last step. The traditional Hamming Distance matching method and HDDMA are compared in the experiment.

\subsubsection{Typical contrast preparations:}

The CASIA-V1.0 iris database is used to show how we do the contrast work. It is suitable to do the feature extracting, encoding and matching work. To analysis the HDDMA we use database A including noise and database $\mathrm{B}$ without noise or with low noise to do the compare experiment. Here database A include 756 images (from 108 eyes) and database B include 490 images (from 70 eyes). We named them noisy iris database A and low-noisy iris database B. For noise iris database A the total matching number is 285390 , inter-class matching number is 2268 , and intra-class matching number is 283122 . For low-noise iris database B the total matching number is 119805 , inter-class matching number is 1470 , and intra-class matching number is 118335 .

We used develop tools Visual C++6.0 and open CV. Computer configuration is as below: Intel i5 3230@2.6GHz CPU and 4G RAM.

In general we use parameters FRR (False Rate of Reject), FAR(False Accept Rate), EER( Equal Error Rate), CRR(Correct Recognition Rate) and ROC curve to evaluate the algorithm performance. EER is enough to evaluate the algorithm performance if the matching method has similar inter-class distribution and intra-class distribution. But from Figure 9 below our HDDMA has different inter-class distribution and intra-class distribution. And FAR and FRR curve is not symmetry in Figure 10. CRR should be considered as a plus to evaluate the algorithm performance.

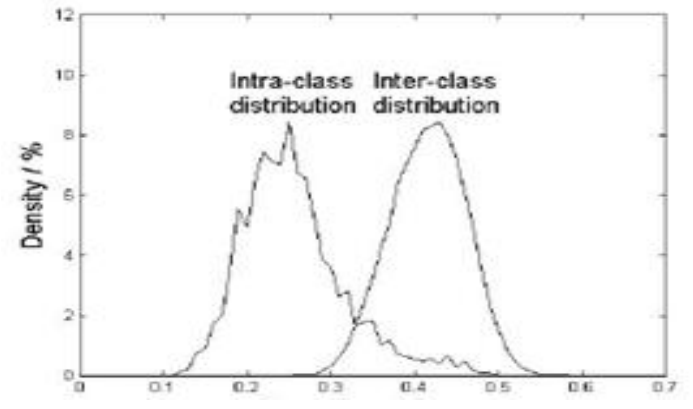

Hamming Distance method

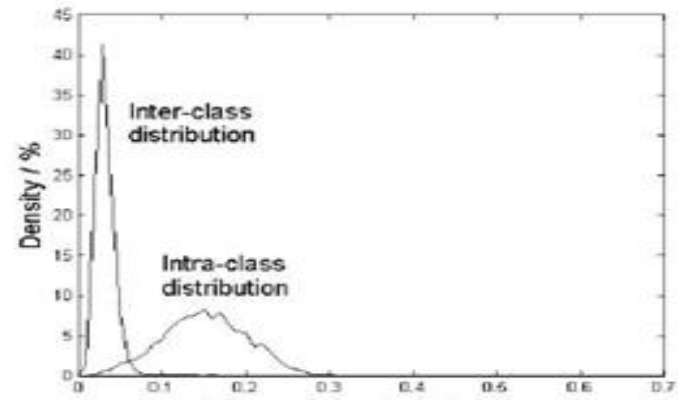

HDDMA

(a) Noisy iris database A 

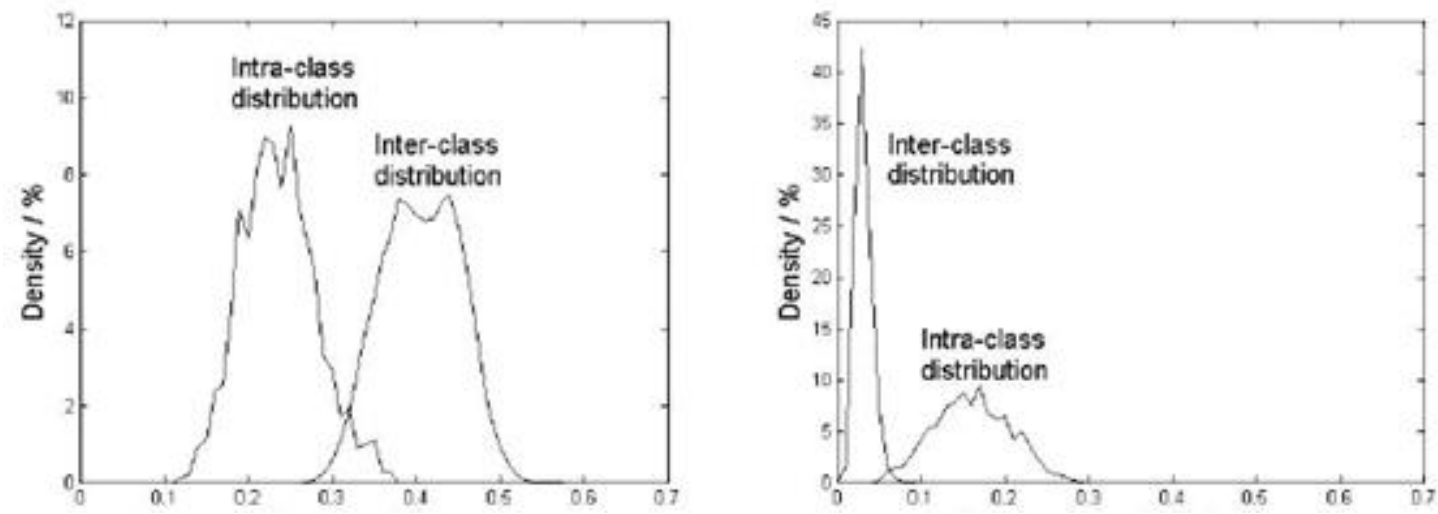

(b) Low-noisy iris database B

Figure 9. Inter-class and Intra-class Distribution Histogram
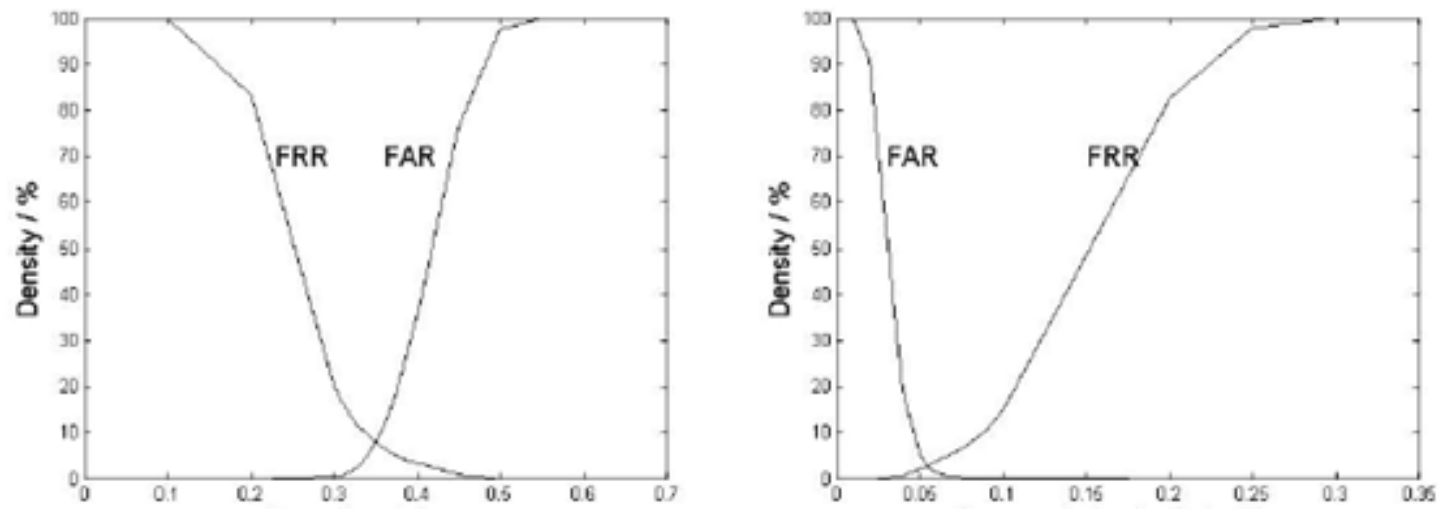

(a) Noisy iris database A
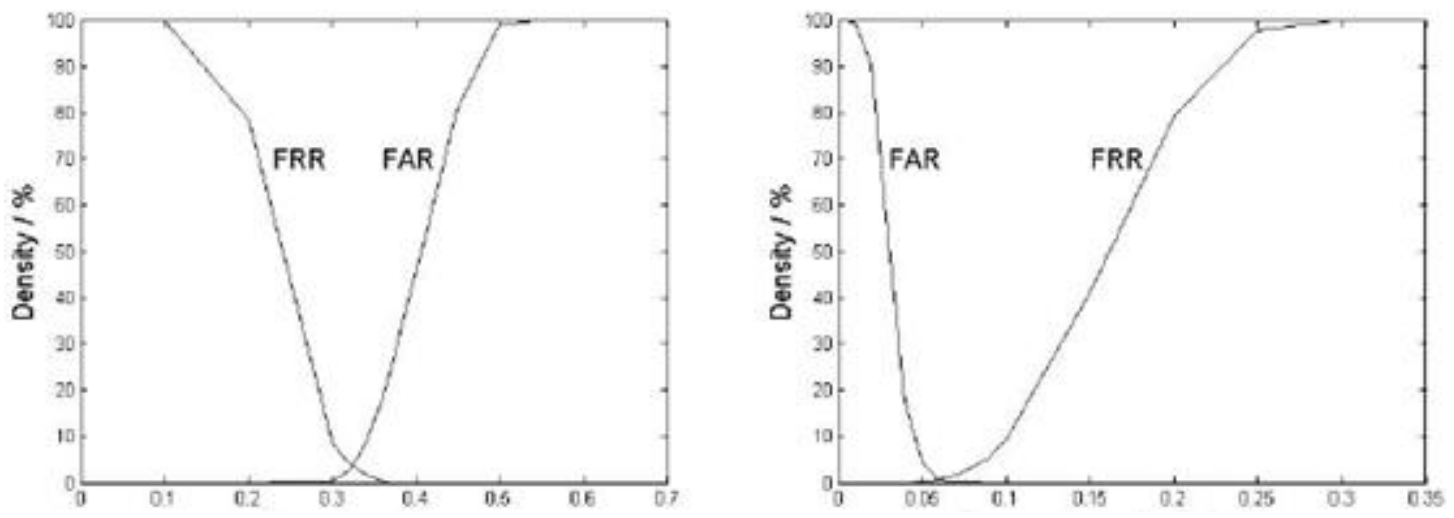

(b) Low-noisy iris database $\mathrm{B}$

Figure 10. FRR and FAR ROC Curve

The first step is pre-processing, the second step is feature extraction, the third step is the iris encoding, and the last step is the match. Here we focused on the last step. The traditional Hamming Distance matching method and HDDMA are compared in the experiment. 


\subsubsection{Recognition Ratio Contrast:}

We list the experiment result in Table I. EER and CRR with HDDMA are better than those with traditional Hamming Distance matching method in the 5 databases. HDDMA shows a significant improvement in the recognition accuracy. In addition, CASIA-V1.0 and CASIA3.0-Interval have more clear images. They are better to evaluate the algorithm performance. HDDMA get the best performance in these two databases. It proves the superiority of HDDMA in another way.

TABLE I Recognition Ratio Comparison of Two Matching Approach

\begin{tabular}{|c|c|c|c|c|}
\hline & Iris Lib & Eyes/Sample Num & EER/\% & CRR/\% when EER \\
\hline TMM & \multirow{2}{*}{ CASIA-1.0 } & \multirow{2}{*}{$108 / 756$} & 2.62 & 97.38 \\
\hline HDDMA & & & 1.34 & 98.66 \\
\hline TMM & \multirow[t]{2}{*}{ CASIA-2.0-Device1 } & \multirow[t]{2}{*}{$60 / 1200$} & 3.51 & 96.49 \\
\hline HDDMA & & & 2.02 & 97.98 \\
\hline TMM & \multirow[t]{2}{*}{ CASIA-2.0-Device2 } & \multirow[t]{2}{*}{$60 / 1200$} & 5.73 & 94.27 \\
\hline HDDMA & & & 5.60 & 94.40 \\
\hline TMM & \multirow[t]{2}{*}{ CASIA-3.0-Interval } & \multirow[t]{2}{*}{$396 / 2641$} & 2.01 & 97.99 \\
\hline HDDMA & & & 0.96 & 99.04 \\
\hline TMM & \multirow[t]{2}{*}{ CASIA-3.0-Twins } & \multirow[t]{2}{*}{$400 / 3183$} & 5.36 & 94.64 \\
\hline HDDMA & & & 4.35 & 95.65 \\
\hline
\end{tabular}

\subsubsection{Anti-noise Ability Contrast:}

The image in the iris database inevitably will lead into eyelids and eyelashes. FR is also one important parameter to evaluate the algorithm. Lower FR means better performance of the algorithm. We list the intra-class FR (False Reject) number comparison of traditional Hamming Distance matching method and HDDMA. HDDMA gets lower FR than traditional Hamming Distance matching method in all five databases.

TABLE II

False Reject Number Comparison of Two Matching Approach

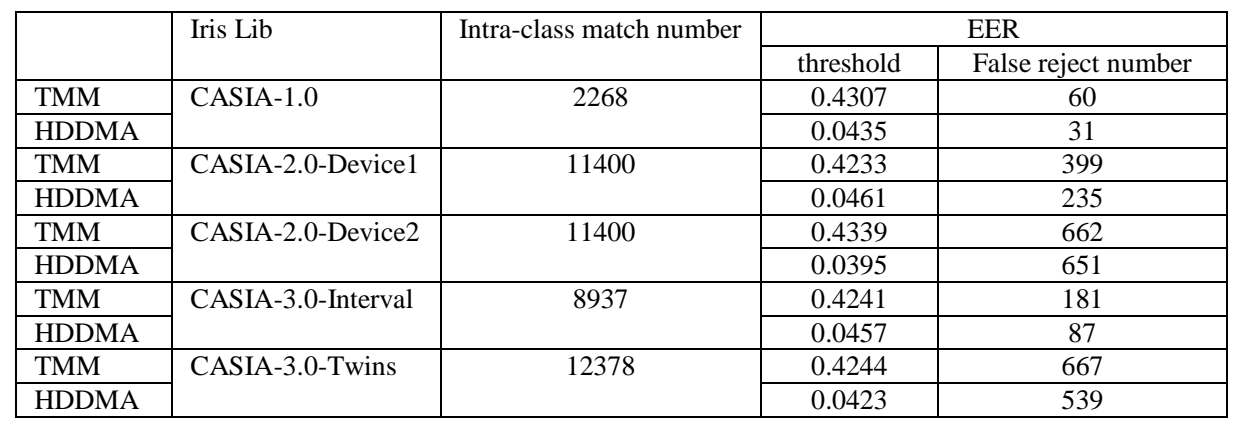

\subsection{Comparison with other Iris Recognition Methods based on Gabor Filter}

We have done the compare experiment in CASIA-V1.0 and listed the result as below: 


\section{TABLE III Recognition Result Comparison of Iris Recognition Methods based on Gabor Filter}

\begin{tabular}{|c|c|c|c|c|c|}
\hline & Eyes/Sample Num & Code/bits & EER/\% & CRR/\% for EER & Highest CRR \\
\hline 8 & $30 / 210$ & 1920 & 1.891 & 98.46 & - \\
\hline 9 & $60 / 420$ & 1536 & 1.990 & 98.31 & - \\
\hline HDDMA & $70 / 490$ & 2048 & 0.748 & 99.23 & 99.949 \\
\hline HDDMA & $108 / 756$ & 2048 & 1.340 & 98.66 & 99.902 \\
\hline
\end{tabular}

HDDMA is used in the complete noisy database CASIA-V1.0 and reduction low-noisy database CASIA-V1.0 respectively. [8, 9] methods only choose the low-noisy images to do the experiments. HDDMA gets better performance even in the complete noisy database CASIA-V1.0. This has fully demonstrated the advantage of HDDMA.

\section{Conclusion}

We use the odd symmetry Gabor filters with single frequency and two directions to extract iris edge features. Especially we raise HDDMA for iris matching. HDDMA consider the whole shift matching process. The main character of HDDMA is the shift curve change. HDDMA can not only get the better matching ratio, but also have strong anti-eyelid and eyelash noise ability. The approach gets the best matching results in CASIA-V3.0-Interval database. The experimental results show that HDDMA is a better iris code matching approach. Traditional Hamming Distance matching method get 99.773\% but HDDMA can get $99.902 \%$ matching ratio in noisy iris database. Traditional Hamming Distance matching method get $99.784 \%$ but HDDMA can get 99.949\% matching ratio in low-noisy iris database.

\section{Acknowledgements}

The funding supports of this work by the Commonweal Project Funds of Zhejiang Science \& Technology Department (Grant No. 2013C31115), the Open Project of Zhejiang Provincial Engineering Center (Grant No. 2012E10023-15) and the Zhejiang Provincial Natural Science Foundation (Grant No. Y13F020205, LY13F020047), are greatly appreciated.

\section{References}

[1] Ms. A. G. Gale1 and Dr. S. S. Salankar, "A Review On Advance Methods Of Feature Extraction In Iris Recognition System”, IOSR Journal of Electrical and Electronics Engineering (IOSR-JEEE) eISSN: 2278-1676, p-ISSN: 2320-3331, (2014), pp. 65-70.

[2] A. S. Alotaibi and M. A. Hebaishy, "Increasing the Efficiency of Iris Recognition Systems by Using MultiChannel Frequencies of Gabor Filter", Journal of Remote Sensing Technology, vol. 2, no. 1, (2014) February, pp. 98-107.

[3] J. G. Daugman, "High Confidence Visual Recognition of Persons by a Test of Statistical Independence", IEEE Trans on Pattern Analysis and Machine Intelligence, vol. 15, no. 11, (1993), pp. 1148-1161.

[4] R. P. Wildes, "Iris Recognition: An Emerging Biometric Technology", Proc of the IEEE, vol. 85, no. 9, (1997), pp. 1348-1363.

[5] W. W. Boles and B. Boashash, "A Human Identification Technique Using Images of the Iris and Wavelet Transform”, IEEE Trans on Signal Processing, vol. 46, no. 4, (1998), pp. 1185-1188.

[6] W. Yunhong, Z. Yong and T. Tieniu, "Biometrics Personal Identification Based on Iris Pattern", Acta Automatica Sinica, vol. 28, no. 1, (2002), pp. 1-10.

[7] M. Li, T. Tieniu, W. Yunhong and D. Zhang, "Efficient Iris Recognition by Characterizing Key Local Variations", IEEE Trans on Signal Processing, vol. 46, no. 4, (1998), pp. 1185-1188. 
[8] G. Yongcai, G. Jianqing, G. Chao and X. Meixuan, "Encoding Algorithm for Iris Image Based on Gabor Filtering", Opto-Electronic Engineering, vol. 33, no. 4, (2006), pp. 35-38.

[9] Y. Weiqi, L. Wanglan and K. Li, "The Parameter Selection of Gabor Filter for Iris Feature Extraction", Journal of Optoelectroincs. Laser, vol. 19, no. 9, (2008), pp. 1235-1239.

[10] Z. Jilin, L. Enyi, W. Jian, R. Yongjian, Y. Miao and W. Jue, "Implementing Sparse Matrix-Vector Multiplication with QCSR on GPU”, Applied Mathematics \& Information Sciences, vol. 7, no. 2, (2013), pp. 473-482.

\section{Authors}

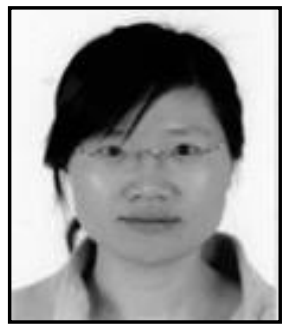

Lv Hanfei, She is an associate professor of Department of Information Technology and Management, Zhejiang Policy Vocational Academy. Before joining Zhejiang Policy Vocational Academy, she was a master student of Zhejiang University from 2002 to 2005. Her current research area include image processing, power aware computing system and its applications in computing systems.

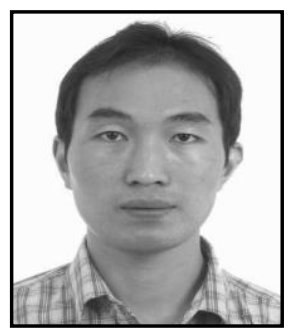

Congfeng Jiang, he is an associate professor of School of Computer Science and Technology, Hangzhou Dianzi University, China. He is with the Grid and Services Computing Lab in Hangzhou Dianzi University. Before joining Hangzhou Dianzi University, he was a $\mathrm{PhD}$ candidate in Huazhong University of Science and Technology from 2002 to 2007. He received his PhD degree in 2007. His current research areas include power aware computing system, virtualization, grid computing, etc., Dr. Jiang is a member of ACM, IEEE, and China Computer Federation (CCF). 
International Journal of Security and Its Applications Vol.8, No.5 (2014) 